\title{
Riesgo individual y poblacional en infarto agudo del miocardio: Estudio IN TERH EART Chile
}

\author{
Fernando Lanas ${ }^{1}$, Sergio Potthoff ${ }^{2}$, Enrique Mercadal ${ }^{3}$, \\ Claudio Santibáñez ${ }^{4}$, Alejandra Lanas ${ }^{1}$, Dina Standen ${ }^{1 a}$. \\ Individual and population risk \\ in acute myocardial infarction: \\ The Chilean INTERHEART study
}

Background: Acute myocardial infarction (AMI) is the first cause of death in Chile. Aim: To assess the magnitude of risk of individuals and population associated with AMI risk factors. Material and methods: Case control study with incident cases and 2 controls paired by age and gender. History of diabetes, hypertension, smoking, stress, depression, diet, weight, height, hip and waist circumference, apolipoprotein (Apo) A1 and B were determined. Odds ratio (OR) and population attributable risk (PAR) were calculated with 95\% confidence interval. Results: Three hundred thirty two cases and 672 controls were included. Mean age was $61.6 \pm 12$ years and $22 \%$ were women. The higher individual risk was associated with smoking: OR 3.1 (2.3-4.2), hypertension: 2.9 (2.1-3.9), permanent stress: 2.2 (1,3-2,8), increased apoB/ApoA1 ratio: 2.1 (1.43.0) and diabetes: 2.0 (1.4-2.9). A protective effect of daily consumption of vegetables and/or fruits with and OR of 0.54 (0.4-0.8), was observed. The highest PAR was due to smoking: 42\% (33.2-51.4), increased ApoB/ApoA1 ratio: 35.2 (19.0-55.8) and hypertension: 32\% (24.5-40.8). These three factors explained $71.3 \%$ of the AMI risk in Chile. A moderate effect on PAR was observed for abdominal obesity: $16.6 \%$ (2.4-61.2), permanent stress: $12.0 \%$ (2.3-44.1) and diabetes: $10.8 \%$ (6.118.3). Conclusions: Known risk factors like dyslipidemia, smoking and hypertension explain most of the AMI cases in Chile. The control of these risk factors should have a major effect on morbidity and mortality due to coronary artery disease in our country (Rev Méd Chile 2008; 136: 555-60).

(Key words: Coronary artery disease; Dyslipidemias; Myocardial infarction)

\begin{abstract}
Recibido el 18 de julio, 2007. Aceptado el 26 de diciembre, 2007.
Financiamiento: Universidad de La Frontera, Proyecto DIUFRO Convenio de desempeño, Sociedad Chilena de Cardiología, Filial Sur. No tuvieron influencia en ninguna etapa del estudio. ${ }^{1}$ Departamento de Medicina Interna y CIGES (Centro Investigación, Gestión y Educación para la Salud) Universidad de La Frontera, Temuco, Chile. ${ }^{2}$ Hospital Base de Osomo. ${ }^{3}$ Hospital Regional de Talca. ${ }^{4}$ Hospital de Castro.

aEnfermera Universitaria
\end{abstract}

L as enfermedades del sistema circulatorio son la primera causa de muerte en Chile, correspondiendo en 2003 a $27 \%$ de la mortalidad total; dentro de las enfermedades cardiovasculares la

Correspondencia a: Dr. Fernando Lanas. Facultad de Medicina, Universidad de La Frontera. M. Montt 112, oficina 306, Temuco. Teléfono: 45 325745. Fax: 45325741.

E mail: flanas@ufro.cl cardiopatía coronaria y el IAM son predominantes ${ }^{1}$. Existen estudios nacionales de corte transversal sobre los factores de riesgo de cardiopatía coronaria a nivel poblacional ${ }^{2-5}$ y en grupos específicos de la población ${ }^{6,7}$. Todas las publicaciones coinciden en una alta frecuencia de estos factores, por ejemplo, la Encuesta Nacional de Salud (ENS) de 2003 muestra que más de la mitad de la población adulta chilena tiene un riesgo cardiovascular alto o muy alto ${ }^{2}$. 
También existe importante información sobre los factores de riesgo en los individuos hospitalizados por IAM y su pronóstico inmediato, fundamentalmente basada en series clínicas como el registro GEM ${ }^{8-10}$. No existe información nacional sobre el riesgo de IAM que produce cada factor de riesgo en forma aislada 0 asociada a otros factores, o del porcentaje de eventos en la población debidos a determinado factor de riesgo estimado por el nesgo atribuible poblacional (RAP).

El estudio INTERHEART es un estudio de casos y controles multinacional, diseñado para evaluar si los factores de riesgo convencional o emergentes tienen una asociación similar con IAM en poblaciones de diferente etnia, estilo de vida y nivel socioeconómico ${ }^{11-16}$. Cerca de 15.000 casos de primer IAM y un número similar de controles fueron incluidos en 52 países. Un objetivo secundario fue estimar el RAP de factores de riesgo aislados o en conjunto en la población total de estudio, en diferentes regiones y en grupos predefinidos. Esta publicación presenta los resultados en los individuos incluidos en el estudio INTERHEART en Chile.

\section{MÉTodos}

Los métodos usados en el estudio INTERHEART han sido publicados en forma detallada en forma previa $^{11,12}$.

Diseño del estudio. Estudio de casos y controles, con casos incidentes de un primer IAM y controles pareados en cada centro por sexo y edad, con un rango de 5 años.

Participantes. Los participantes del estudio fueron reclutados en hospitales de Temuco, Talca, Osorno y Castro. Casos: Pacientes con un primer IAM ingresados en las primeras $24 \mathrm{~h}$ del comienzo de síntomas, con cambios electrocardiográficos caracteństicos de IAM y confirmados posteriormente por elevación de enzimas. Los pacientes fueron excluidos si estaban en shock cardiogénico, tenían historia previa de enfermedad cardíaca o una enfermedad médica crónica significativa que pueda modificar los factores de niesgo. Controles: Los controles tenían los mismos criterios de exclusión de los casos. Los controles podían ser individuos hospitalizados por una enfermedad no relacionada con los factores de riesgo del IAM, o ser visitas de un paciente que no presentara enfermedad cardíaca. El protocolo permitía reclutar uno o dos controles por caso y en Chile se incluyeron 2 controles por caso para incrementar el poder estadístico.

Procedimientos. Se obtuvo información con un cuestionario estructurado sobre factores demográficos, socioeconómicos (educación, ingreso), estilo de vida (tabaco, actividad física, dieta), factores psicosociales (depresión, estrés percibido, eventos estresantes y locus de control) historia personal y familiar de enfermedad cardiovascular y factores de riesgo (hipertensión, diabetes mellitus). Se determinó peso, talla, circunferencia de cintura y cadera, pulso y presión y se tomó una muestra de sangre. Para el análisis de lípidos sanguíneos se usó la relación de apolipoproteína (Apo) B/ Apo A1.

Se definió como fumador actual a quien ha fumado cualquier cantidad en los últimos 12 meses y como ex fumador a quien había dejado de fumar al menos el último año, los terciles de corte de la relación de peńmetro cintura/cadera fueron derivados de la muestra total de controles del estudio INTERHEART: 0,90 y 0,95 en hombres y 0,83 y 0,90 en mujeres. Se consideraron físicamente activos a los individuos que realizaban ejercicio moderado (ej: caminar, trabajar en el jardín, andar en bicicleta) o intenso (ej: trotar, nadar, jugar fútbol) a lo menos $4 \mathrm{~h}$ a la semana. El consumo regular de alcohol fue definido como el consumo 3 o más veces a la semana. Estrés global fue definido como estrés reportado en casa o trabajo. El protocolo fue aprobado por el Comité de Ética Científico del Servicio de Salud Araucanía Sur. Los participantes firmaron consentimiento informado.

Estadística. Los promedios se muestran con su desviación estándar en paréntesis. Las asociaciones para proporciones fueron evaluadas con el test de $\chi^{2}$ de Pearson y Tablas de contingencia y para variables continuas con test de Student. Los puntos de corte para separar la muestra en terciles o quintiles fueron derivados de la población total de controles de INTERHEART. Para los factores protectores (dieta, alcohol, ejercicio) el RAP fue calculado para el grupo sin la exposición. El odds ratio $(\mathrm{OR})$ para tabaquismo fue ajustado por edad y sexo, los otros OR fueron ajustados por tabaquismo, edad y sexo. Para estimar el efecto de cada factor en forma independiente se utilizó regresión logística no condicional con ajuste por los factores de pareamiento. Este procedimiento 
dio resultados similares a la regresión logística condicional y además permite incluir casos en que el pareamiento no fue posible. Los OR estimados y su intervalo de confianza de $95 \%$ se presentan para cada factor de riesgo y combinaciones de ellos. El RAP fue calculado usando la fórmula habitual para exposición y enfermedad simple y dicotómica ${ }^{17}$. El análisis estadístico se realizó con SAS versión 8.2 (SAS, Cary, NC, USA) y S-Plus versión 6 (Insightful, Seattle, WA, USA).

\section{Resultados}

Entre febrero de 1999 y marzo de 2003, se reclutaron 322 casos y 672 controles en Chile. De los casos y controles, 78\% eran hombres y la edad promedio de los casos fue $61,6 \pm 12,1$ años, en los hombres fue $60,7 \pm 12,3$ y en las mujeres fue $64,9 \pm 10,7$ ( $p<0,01$ ). La prevalencia de factores de riesgo en los controles y el OR Chile, América Latina y el total del estudio INTERHEART, excluyendo América Latina se muestra en la Tabla 1, los resultados del RAP para los mismos grupos se muestran en la Tabla 2.

Prevalencia de factores de riesgo en los controles (Tabla 1). Los factores de riesgo más frecuentes en el grupo control, afectando a más de un tercio de esa población, fueron el índice cintura cadera aumentado, niveles anormales de apolipoproteínas y tabaquismo, aunque la mitad de estos últimos eran ex fumadores.
La frecuencia de depresión también fue elevada. Entre los factores protectores $87,7 \%$ de los controles consumía diariamente frutas o verduras y $25,1 \%$ hacia actividad física en forma regular. El 47,3\% reportaba algunos periodos de estrés, $11,4 \%$ varios periodos y en $4,7 \%$ el estrés reportado era permanente.

Fuerza de asociación (Tabla 1). El consumo presente o pasado de tabaco tuvo el mayor riesgo, medido como OR, seguido del antecedente de hipertensión arterial y la presencia de estrés permanente. El riesgo asociado a fumar fue más alto en fumadores actuales, OR 3,46 (2,41-4,97), alcanzando a 7,19 $(3,91-13,23)$ en fumadores de más de 20 cigarrillos/día. El estrés percibido también tuvo una relación exposición-riesgo, con OR de 1,03 (0,74$1,44)$ para aquellos que reportaron estrés ocasional, $1,45(0,9-2,34)$ para quienes relataban varios periodos de estrés y 2,19 $(1,27-3,78)$ para estrés permanente. El índice de masa corporal, al comparar el tercil medio con el inferior, tuvo un OR de 1,42 $(0,9-2,2)$ y el tercil superior, en relación al inferior tuvo un OR de 1,95 (1,2-3,0). Hubo un aumento de riesgo al doble en quienes estaban en el tercil superior de la relación ApoB/ApoA1 y en los que tenían historia personal de diabetes mellitus. La actividad física regular y el consumo de frutas 0 verduras a diario redujeron el riesgo en $18 \%$ y $46 \%$, respectivamente. No hubo asociación entre riesgo de IAM y depresión, consumo de alcohol e índice cintura cadera.

Tabla 1. Prevalencia de factores de riesgo de infarto agudo del miocardio en Chile, América Latina y el estudio INT ERH EART total, excluyendo América Latina y su 0 dds Ratio e intervalo de confianza de $95 \%$

\begin{tabular}{|c|c|c|c|c|c|c|}
\hline \multirow[t]{2}{*}{ Factor de riesgo } & \multicolumn{3}{|c|}{ Prevalencia en controles (\%) } & \multicolumn{3}{|c|}{ Odds Ratio (95\% IC) } \\
\hline & Chile & $\mathrm{AL}$ & IH-AL & Chile & $\mathrm{AL}$ & IH-AL \\
\hline ApoB/ApoA1* & 42,3 & 42 & 32 & $2,05 \quad(1,4-3)$ & $2,31 \quad(1,83-9,4)$ & $(2,8-3,3)$ \\
\hline Tabaco** & 36,5 & 48,1 & 48,1 & $3,1 \quad(2,3-4,2)$ & $2,31 \quad(1,97-2,71)$ & $2,26 \quad(2,1-2,4)$ \\
\hline Diabetes mellitus & 11,7 & 9,54 & 7,2 & $(1,4-2,9)$ & $2,59 \quad(2,09-3,22)$ & $3,16 \quad(2,9-3,49)$ \\
\hline Hipertensión arterial & 26,7 & 29,1 & 20,8 & $2,86(2,1-0,9)$ & $2,81 \quad(2,39-3,31)$ & $2,41 \quad(2,3-2,6)$ \\
\hline Relación cintura-cadera* & 48,9 & 48,6 & 31,2 & $1,26 \quad(0,8-, 9)$ & $2,49(1,97-3,14)$ & $2,22 \quad(2,1-2,4)$ \\
\hline Depresión & 41 & 28,9 & 15,8 & $0,95 \quad(0,7-3)$ & $1,17 \quad(0,98-1,38)$ & $(1,5-1,7)$ \\
\hline Estrés permanente**** & 4,7 & 6,8 & 3,9 & $2,19(1,3-0,8)$ & $2,81 \quad(2,07-3,82)$ & $(1,8-2,4)$ \\
\hline Ejercicio regular & 25,1 & 22 & 18,9 & $0,82(0,6-1,2)$ & $0,67 \quad(0,55-0,82)$ & $0,7 \quad(0,65-0,76)$ \\
\hline Alcohol & 16,2 & 19,4 & 11,9 & $1,2 \quad(0,8-1,7)$ & $1,05 \quad(0,86-1,27)$ & $0,78(0,74-0,84)$ \\
\hline Frutas 0 verduras diariamente & 87,7 & 85 & 83,3 & $0,54 \quad(0,4-0,8)$ & $0,69(0,57-0,83)$ & $0,78(0,73-0,84)$ \\
\hline
\end{tabular}

IH-AL: Participantes en el estudio INTERHEART excluyendo América Latina, AL: América Latina $*^{\text {st }}$ vs $3^{\text {rd }}$ tercil, **Nunca vs actuales y ex-fumadores, ***Nunca vs permanente. 


\section{Tabla 2. Riesgo atribuible poblacional de infarto agudo del miocardio en Chile, América Latina y el estudio IN TERH EART total, excluyendo América Latina, e intervalo de confianza de $95 \%$}

\begin{tabular}{|lllrlrr|}
\hline Factor de riesgo & \multicolumn{5}{c|}{ Riesgo atribuible poblacional (95\% CI) } \\
& \multicolumn{2}{c}{ Chile } & \multicolumn{2}{c|}{ AL } & & IH-AL \\
\hline ApoB/ApoA1* & 35,2 & $(18,9-55,8)$ & 40,8 & $(30,3-52,2)$ & 44,2 & $(41,3-47,1)$ \\
Tabaco** & 42 & $(33,2-51,4)$ & 38,4 & $(32,8-44,4)$ & 35,3 & $(33,3-37,4)$ \\
Diabetes mellitus & 10,8 & $(6,1-18,3)$ & 12,9 & $(10,3-16,1)$ & 12,2 & $(11,3-13,1)$ \\
Hipertensión arterial & 32 & $(24,5-40,8)$ & 32,9 & $(28,7-37,5)$ & 22,0 & $(20,7-23,4)$ \\
Relación cintura-cadera* & 16,6 & $(2,4-61,2)$ & 45,8 & $(35,8-56,2)$ & 30,2 & $(27,4-33,2)$ \\
Depresión & $-2,2$ & $(-13-8,7)$ & 4,7 & $(1,4-13,9)$ & 8,4 & $(7,3-9,7)$ \\
Estrés permanente*** & 12,0 & $(2,3-44,1)$ & 28,1 & $(18,5-40,3)$ & 7,8 & $(4,6-13,1)$ \\
Ejercicio regular & 14,7 & $(2,6-52,3)$ & 28,0 & $(17,7-41,3)$ & 24,8 & $(20,6-29,6)$ \\
Alcohol & -16 & $(-50-18,4)$ & $-3,2$ & $(-18-11,7)$ & 16,3 & $(12,7-20,6)$ \\
Frutas o verduras diariamente & 12,1 & $(6,1-18,1)$ & 6,9 & $(3,35-10,5)$ & 4,1 & $(2,9-5,3)$ \\
\hline
\end{tabular}

IH-AL: Participantes en el estudio INTERHEART excluyendo América Latina, AL: América Latina. $*_{1}^{\text {st }}$ vs $3^{\text {rd }}$ tercil, **Nunca vs actuales y ex-fumadores, ***Nunca vs permanente.

Para los factores protectores (dieta, ejercicio, y alcohol) el RAP corresponde al grupo sin esos factores.

Riesgo atribuible poblacional (Tabla 2). Los 3 factores que determinaron un mayor riesgo de IAM en la población fueron el tabaquismo, relación elevada de Apo B/Apo A1 e hipertensión arterial. El RAP conjunto de estos 3 factores fue $71,3 \%(61 \%$ $81,7 \%$ ) Un riesgo poblacional menor, entre $10 \%$ y $20 \%$, se observó para obesidad abdominal, diabetes mellitus, ausencia de ejercicio regular o de consumo diario de frutas o verduras.

Sexo. Las diferencias más marcadas en prevalencia de factores de riesgo entre hombres y mujeres en el grupo control se observaron para el consumo de tabaco, siendo más de 2 veces más frecuente en hombres y para el consumo regular de alcohol, 6 veces más frecuente en hombres. La depresión, obesidad abdominal, historia de hipertensión arterial y diabetes mellitus fueron más frecuentes en mujeres.

La estimación de la magnitud del riesgo, usando OR, asociado a los factores de riesgo estudiados fue en general muy similar entre hombres y mujeres, observándose mayor riesgo en hombres con obesidad abdominal (OR $=2,25$ en hombres y 1,12 en mujeres) y en el efecto del estrés permanente (OR $=2,41$ en hombres y 1,73 en mujeres). En las mujeres hubo mayor riesgo asociado a diabetes mellitus (OR $=1,76$ en hombres y 2,62 en mujeres).

\section{Discusión}

Nuestro estudio demuestra que los 3 factores de riesgo que determinan la mayor parte del riesgo de
IAM en Chile son el consumo de tabaco, un nivel inadecuado de lípidos sanguíneos y la hipertensión arterial. La diabetes mellitus, obesidad abdominal y estrés permanente tuvieron un efecto menor. El consumo de frutas y verduras tuvo un importante efecto protector, hubo una tendencia a un efecto protector del ejercicio que no logró significación estadística y no se observó asociación de depresión y consumo de alcohol con IAM.

La alta frecuencia de consumo de tabaco en Chile ha sido reportada en estudios previos de prevalencia ${ }^{2-}$ 7; en la ENS de 2003 hubo 34,4\% de tabaquismo en el rango de edad de 45-64 años, el que más se aproxima a la edad de los individuos de nuestro estudio. Las proyecciones de la Organización Mundial de Salud estiman que para el año 2020 se convertirá en la mayor causa se muerte a nivel mundial, siendo responsable de $12,3 \%$ de las muertes en el mundo ${ }^{18}$. En nuestro estudio, el riesgo individual y poblacional más alto se asoció al consumo de tabaco, a diferencia de lo que ocurrió en América Latina ${ }^{16} 0$ el resto de los países del estudio INTERHEART ${ }^{11}$, en que el riesgo atribuible poblacional más alto se debió a obesidad abdominal y niveles inapropiados de Apo B/Apo A1, respectivamente. La dislipidemia es frecuente en la población general en Chile, en la ENS más de $50 \%$ de las personas entre 45 y 64 años tenían niveles elevados de colesterol ${ }^{2}$. Estos resultados son difíciles de comparar con los de nuestro grupo control, dado que en nuestro estudio se determinó la relación de apolipoproteínas y se usó como comparación los 
datos de los controles en la muestra total del estudio. La determinación de apolipoproteínas tiene un mayor poder predictivo que la determinación de lipoproteínas ${ }^{19-22}$ y además no está influida por el estado de ayuno o no.

La prevalencia de hipertensión conocida en el grupo control, 26,7\%, es más baja que la tasa observada en el rango de edad de 45 a 64 años en la ENS, 53,7\%2. Sin embargo, esta misma encuesta demuestra que sólo $60 \%$ de los hipertensos conoce su condición (32\% de la muestra total). En otros estudios nacionales, la prevalencia de hipertensión reportada es más baja y se acerca a nuestros dato ${ }^{3-6}$, sin embargo, la comparación entre estudios está limitada por las caracteństicas de la muestra y los criterios diagnósticos de cada estudio. La importancia de la hipertensión en Chile y América Latina es mayor que en el resto del mundo. Se puede postular que las altas tasas de hipertensión arterial y diabetes mellitus en nuestra región están asociadas a una mayor obesidad abdominal, lo que ya habíamos reportado en el estudio de factores de riesgo en países en desamollo INCLEN $^{23}$ y se relaciona con el fenómeno de transición epidemiológica observado en nuestro país y región ${ }^{24-25}$.

La frecuencia de estrés fue más elevada en América Latina y el riesgo asociado de IAM al presentar estrés permanente es similar a estar en el tercil superior de la relación ApoB/Apo A1 o a ser diabético. Sin embargo, no hubo asociación entre depresión e IAM, mientras que en el estudio INTERHEART considerando todos los países hubo un mayor riesgo de $60 \%$. Nuestros resultados deben ser interpretados considerando las limitaciones y posibles sesgos de un estudio de casos y controles, en que la presencia de variables de exposición se determina luego de la aparición de la enfermedad. Pese a la dificultad de medir variables como estrés y depresión, y la posibilidad de sesgos al estimarla, existe clara evidencia en la literatura de su asociación con IAM ${ }^{26-}$ 28. Esta diferencia puede deberse a una tendencia a

\section{REFERENCIAS}

1. www.ine.cl. Anuario de estadísticas vitales 2003, página 358.

2. epi.minsal.cl/epi/html/invest/ENS/ENS.htm Encuesta Nacional de Salud 2003. Ministerio de Salud.

3. Berríos X, Jadue L, Zenteno J, Ross MI, Rodríguez $H$. Prevalencia de factores de riesgo de enferme- no reportar síntomas depresivos en los hombres con IAM o puede ser el resultado del azar, lo que es esperable al hacer análisis de subgrupos.

El sedentarismo es prácticamente la conducta habitual en Chile, con prevalencia superior a 90\% para hombres y mujeres ${ }^{2}$. En nuestro estudio un porcentaje más alto se consideró como físicamente activo, lo que es explicado por una manera diferente de dicotomizar los resultados. Habitualmente se define como activo al que hace más de 30 min 3 veces a la semana de ejercicio, pero en nuestro estudio determinamos la actividad física en el hogar, trabajo y esparcimiento, sumando las horas de actividad moderada e intensa, lo que explica las diferencias con publicaciones previas. Una proporción muy alta de individuos consume frutas 0 verduras en forma diaria, lo que se asoció a una importante reducción de riesgo individual: $\mathrm{OR}=0,54$, sin embargo dado que sólo $12,3 \%$ de los individuos no consumía diariamente frutas o verduras el riesgo atribuible poblacional por esta causa es bajo. En nuestros resultados en Chile el consumo de alcohol no tuvo un efecto protector, a diferencia de lo observado en el estudio INTERHEART ${ }^{11}$ en la totalidad de sus individuos o el estudio AFFIRMAR, hecho en Brasil ${ }^{29}$. Esto pudiera relacionarse con patrones específicos del beber en Chile (tipo de bebida alcohólica o intensidad de consumo), a la forma de dicotomizar los resultados, de modo que los bebedores de menos de 3 veces a la semana se analizan junto con los no bebedores, o ser producto del azar en el análisis de subgrupos.

En resumen, nuestros datos muestran el rol de los factores de riesgo en el IAM en Chile, con un marcado efecto del tabaquismo, de un perfil de lípidos anormal y de la hipertensión arterial, que explican la mayor parte de los casos en el país. Una estrategia de control de estos factores de riesgo, más una promoción de mayor ejercicio y actividad física tendrán un impacto sustancial en el control de la enfermedad coronaria.

dades crónicas. Estudio de población general de la Región Metropolitana 1986-1987, Rev Méd Chile 1990; 118: 597-604.

4. Vega J, Jadue L, Escobar MC, Jall J, Espejo F, Delgado I ET AL. Prevalencia de hipertensión arterial en Valparaíso. Resultados de la encuesta de base del programa CARMEN. Rev Méd Chile 1999; 127: 729-38.

5. Lanas F, Davis MR, Standen D, Iuesca M, Doghetti J, 
Stockins B. Prevalencia de factores de riesgo en la ciudad de Temuco. Rev Méd Chile 1991; 119: 247-51.

6.- Lanas F, Del Solar J, Maidonado M, Guerrero MA, Espinoza F. Prevalencia de factores de riesgo para enfermedad cardiovascular en un grupo de empleados Chilenos. Rev Méd Chile 2003; 131: 129-34.

7. Dadán S, Aicardi V, Kauffmann R, Bunout D. Factores de riesgo cardiovascular en hombres adultos sanos. Rev Méd Chile 1996; 124: 911-7.

8. GRuPo de Estudios Multicéntrico del InFarTo. Farmacoepidemiología del infarto agudo del miocardio en 33 hospitales chilenos. Rev Chilena Cardiol 1995; 14: 11-9.

9. Corbalán R, Nazzal C, Prieto JC, Chávez E, Lanas F, LAMICH R ET AL. Reducción de la mortalidad por infarto del miocardio en los hospitales chilenos. Rev Méd Chile 2002; 130: 368-78.

10. Prieto JC, Corbalán R, Nazzar C, Chávez E, Lanas F, Bartolucci J et al. Cambios en los patrones de prescripción en el Infarto Agudo del Miocardio. Comparación de 2 períodos. Rev Méd Chile 2001; 129: 481-8.

11. Yusuf S, Hawken S, Ounpuu S, Dans T, Avezum A, LANAS F ET AL. INTERHEART Study Investigators. Effect of potentially modifiable risk factors associated with AMI in 52 countries (the INTERHEART study): case-control study. Lancet 2004; 364: 93752.

12. Ounpuu S, Negassa A, Yusuf S. INTER-HEART: A global study of risk factors for acute myocardial infarction. Am Heart J 2001; 141: 711-21.

13. Rosengren A, Hawken S, Ounpuu S, Suiwa K, Zubaid M, ALMAHMEED WA ET AL. INTERHEART investigators. Association of psychosocial risk factors with risk of acute myocardial infarction in 11119 cases and 13648 controls from 52 countries (the INTERHEART study): case-control study. Lancet 2004; 364: 953-62.

14. Yusuf S, Hawken S, Ounpuu S, Bautista L, Franzosi MG, COMMERFORD P ET AL. INTERHEART Study Investigators. Obesity and the risk of myocardial infarction in 27.000 participants from 52 countries: a case-control study. Lancet 2005; 366: 1640-9.

15. Teo KK, Ounpuu S, Hawken S, Pandey MR, Valentin V, Hunt D ET AL. INTERHEART Study Investigators Tobacco use and risk of myocardial infarction in 52 countries in the INTERHEART study: a casecontrol study. Lancet 2006; 368: 621-2.

16. Lanas F, Avezum A, Bautista L Díaz R, Luna M, Islam $\mathrm{S}$ ET AL. INTERHEART investigators in Latin America risk factors for myocardial infarction in Latin America: The Interheart Latin America study. Circulation 2007; 115: 1067-74.

17. WALTER SD. The distribution of Levin's measure of attributable risk. Biometrika 1975; 62: 371-4.

18. Wordd Health Organization. Tobacco or Health? First Global Status Report. Geneva, Switzerland: World Health Organization; 1996.

19. Waldius G, Jungner I, Holme I, Aastveit AH, Kolar W, STEINER E. High apolipoprotein B, low apolipoprotein A-I, and improvement in the prediction of fatal myocardial infarction (AMORIS study): a prospective study. Lancet 2001 358: 2026-33.

20. WALDIUS G, JungNER I. Rationale for using apolipoprotein B and apolipoprotein A-1 as indicators of cardiac risk and as target for lipid lowering therapy. Eur Heart J 2005; 26: 210-2.

21. Meisinger C, Loewel H, Mraz W, Koenig W. Prognostic value of apolipoprotein $B$ and A-I in the prediction of myocardial infarction in middle-aged men and women: results from the MONICA/KORA Augsburg cohort study. Eur Heart J 2005; 26: 271-8.

22. Lamarche B, Moorjani S, Lupien PJ, Cantin B, Bernard PM, Dagenais GR et al. Apolipoprotein A-I and B levels and the risk of ischemic heart disease during a five-year follow-up of men in the Quebec cardiovascular study. Circulation 1996; 94: 273-8.

23. Inclen Multicentre Colaborative Group. Risk factors for cardiovascular disease in the developing world. A multicentre collaborative study in the International Clinical Epidemiology Network (INCLEN). J Clin Epidemiol 1992; 45: 841-7.

24. Albala C, Vio F, Kain J, Uauy R. Nutrition transition in Latin America: the case of Chile. Nutr Rev 2001; 59: 170-6.

25. York DA, Rossner S, Caterson I, Chen CM, James WP, Kumanyika $S$ et al. American Heart Association. Prevention Conference VII: Obesity, a worldwide epidemic related to heart disease and stroke: Group I: worldwide demographics of obesity. Circulation 2004; 110: e463-70.

26. HemingwaY H, Marmot M. Evidence based cardiology: psychosocial factors in the aetiology and prognosis of comnary heart disease: systematic review of prospective cohort studies. BMJ 1999; 318: 1460-7.

27. Kivimaki M, LEino-Arjas $P$, LuUKKonen R, Rithimaki $H$, VAHTERA J, Kirjonen J. Work stress and risk of cardiovascular mortality: prospective cohort study of industrial employees. BM 2002; 325: 857-61.

28. Anda R, Wimamson D, Jones D, Macera C, Eaker E, Glassman A ET AL. Depressed affect, hopelessness, and the risk of ischemic heart disease in a cohort of US adults. Epidemiology 1993; 4: 285-94.

29. Piegas L, Avezum A, Pereira JL, Rossi Neto J, Hoepfener C, FARRAN J ET AL. On behalf of the AFIRMAR Study Investigators. Risk factors for myocardial infarction in Brazil. Am Heart J 2003; 146: 331-8. 